\title{
Effect of High Pressure on the Molecular Structure and $\pi$-Electrons Delocalization of Canthaxanthin as Revealed by Raman Spectra
}

\author{
Shun-li Ou-Yang, ${ }^{1}$ Nan-nan Wu, ${ }^{1,2}$ and Yan-jie Tian ${ }^{3}$ \\ ${ }^{1}$ Key Laboratory of Integrated Exploitation of Bayan Obo Multi-Metal Resources, Inner Mongolia University of Science \& Technology, \\ Baotou 014010, China \\ ${ }^{2}$ School of Mathematics, Physics and Biological Engineering, Inner Mongolia University of Science \& Technology, Baotou 014010, China \\ ${ }^{3}$ Department of Fundamental Physics, College of Science, China University of Petroleum, Qingdao, Shandong 266555, China
}

Correspondence should be addressed to Nan-nan Wu; woshinannan04@imust.cn

Received 30 May 2015; Revised 27 July 2015; Accepted 10 August 2015

Academic Editor: Christoph Krafft

Copyright (C) 2015 Shun-li Ou-Yang et al. This is an open access article distributed under the Creative Commons Attribution License, which permits unrestricted use, distribution, and reproduction in any medium, provided the original work is properly cited.

\begin{abstract}
The effect of high pressure on the molecular structure and $\pi$-electron delocalization of canthaxanthin was studied by in situ resonance Raman spectroscopy. Changes in the characteristic band frequency and the pressure of canthaxanthin were described. The effect of pressure on $\pi$-electron delocalization was also discussed. Results show that the characteristic bands of canthaxanthin increase and reach high wavenumbers. The correlations between Raman frequency of the three main bands and pressure are listed as follows: $v_{1}(\mathrm{C}=\mathrm{C})=3.43 P+1512.3, v_{2}(\mathrm{C}-\mathrm{C})=3.29 P+1156.1$, and $v_{3}\left(\mathrm{CH}_{3}\right)=2.16 P+1006.3$. The frequency multiplication of canthaxanthin changes as pressure is altered. The pressure effect on the $\nu_{1}(\mathrm{C}=\mathrm{C})$ mode is more susceptible than on the $\nu_{2}(\mathrm{C}$-C) mode, which can be explained by the fact that the $\beta$-ring twists to a larger angle from the plane of the conjugated main chain under high pressure, leading to a lower degree of the $\pi$-electrons delocalization. The Raman spectra are recovered after the compressiondecompression cycle indicating the canthaxanthin has no evident phase change under our experimental conditions.
\end{abstract}

\section{Introduction}

Conjugated polyenes are essential photoelectric materials widely applied in physics (nonlinear optical materials), chemistry (molecular optoelectronic devices), and biology (photosynthesis and nanomaterials) [1-7]. These applications are based on the unique molecular structure of conjugated polyenes [8]. Therefore, the molecular structure and the characteristics of conjugated polyenes have been extensively studied $[9,10]$. As an example of conjugated polyenes, canthaxanthin is a linear polyene chain molecule, in which the main chain consists of nine conjugated double bonds (Figure 1).

In nature, the interaction between canthaxanthin and biological macromolecules can change the molecular structure and the electronic state of canthaxanthin [11]. At high pressure, this interaction leads to changes in force constant of molecular bonds, thereby altering molecular structures and electron orbits. These changes are similar to natural changes occurring in canthaxanthin.

Raman spectroscopy, equipped with a diamond anvil cell (DAC) as a high-pressure apparatus, is a powerful tool to investigate the modifications in molecular arrangements and the changes in electron orbits. For instance, Liu et al. applied high-pressure Raman spectroscopy to investigate the changes in characteristic peaks of fundamental frequency as pressure increases from $0 \mathrm{GPa}$ to $5 \mathrm{GPa}$ [12]; on the basis of experimental results, Liu et al. proposed a competition mechanism model and used density function to analyze the effect of $\beta$-ring twisted on both ends of $\beta$-carotene in the Raman spectra [13]. Further, in our studies, Raman spectroscopy has been applied to investigate $\beta$-carotene under high pressure [14]. Likewise, we used a high-pressure DAC and a confocal microscope equipped with Raman spectrometer in our study 


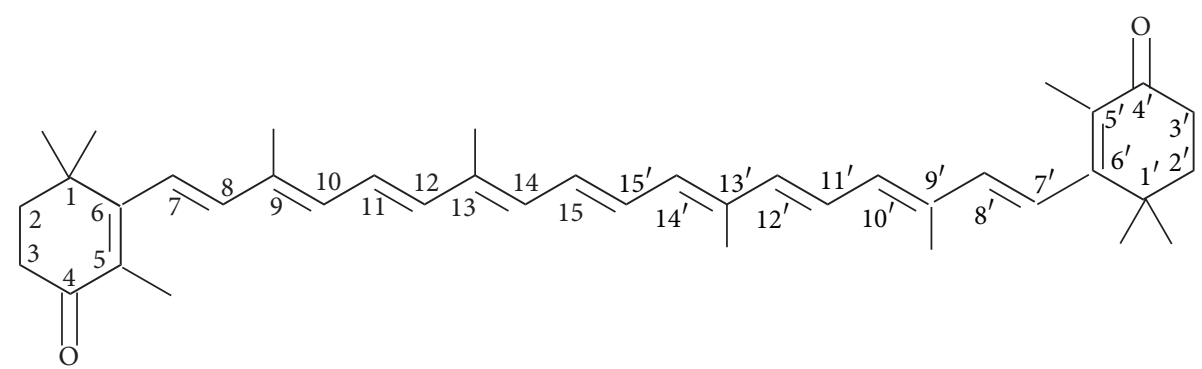

FIGURE 1: Chemical structure of canthaxanthin.

to systematically investigate the structure of canthaxanthin. This study specifically aimed (1) to obtain in situ highpressure Raman spectra of this polyene at pressure ranging from normal to $10.39 \mathrm{GPa},(2)$ to investigate the fundamental frequency observed in the Raman spectra of canthaxanthin, (3) to analyze the Raman spectra of frequency multiplication, and (4) to determine the influence of high pressure on molecular structure and $\pi$-electrons delocalization.

\section{Experimental}

Canthaxanthin was purchased from Sigma, stored at $-18^{\circ} \mathrm{C}$ in the dark, and used without further purification. Canthaxanthin was diluted by the ternary system (Methanol/Ethanol/ Water, $\left.V_{\text {Methanol }}: V_{\text {Ethanol }}: V_{\text {Water }}=16: 3: 1\right)$ at concentration $4.2 \times 10^{-4} \mathrm{M}$. The pressure apparatus used in this experiment is a Mao-Bell diamond anvil cell (DAC) with two diamonds of $0.5 \mathrm{~mm}$ culet size. The liquid sample with a small ruby chip (about $0.1 \mathrm{~mm}$ ) was loaded in a $0.24 \mathrm{~mm}$ hole drilled in a $0.25 \mathrm{~mm}$ thick T301 steel gasket. The pressure calibration was carried out using the Ruby fluorescence.

The micro Raman spectrometer (Renishaw InVia Raman microscope) equipped with $514.5 \mathrm{~nm}$ argon ion $\left(\mathrm{Ar}^{+}\right)$excitation laser (Spectra Physics 163-M142) was used to obtain Raman spectra. Thus, in the present study, we investigate canthaxanthin by means of resonance Raman spectroscopy. The 50x objective was used for both laser illumination and Raman backscattering detection. The excitation power of $6.8 \mathrm{~mW}$ and exposure time (CCD integration time) $12 \mathrm{~s}$ were used to collect all Raman spectra. The resolution of the system was $1.4 \mathrm{~cm}^{-1}$. The experiment was performed at room temperature in a dark room.

\section{Results and Discussion}

3.1. The Resonance Raman Spectrum of Canthaxanthin at Normal Pressure and Assignments. Figure 2 shows the resonance Raman spectrum of canthaxanthin at normal pressure. The Raman spectrum of canthaxanthin is divided into two major parts. The three main bands are found from $950 \mathrm{~cm}^{-1}$ to $1650 \mathrm{~cm}^{-1}$, and these bands can be assigned to the $\mathrm{C}=\mathrm{C}$ in-phase stretching, the $\mathrm{C}-\mathrm{C}$ stretching, and the methyl inphase rocking mode, respectively. The corresponding spectral bands are located at 1515, 1155, and $1005 \mathrm{~cm}^{-1}$ and are called the $v_{1}, v_{2}$, and $v_{3}$ bands in Figure 2. The Raman spectrum

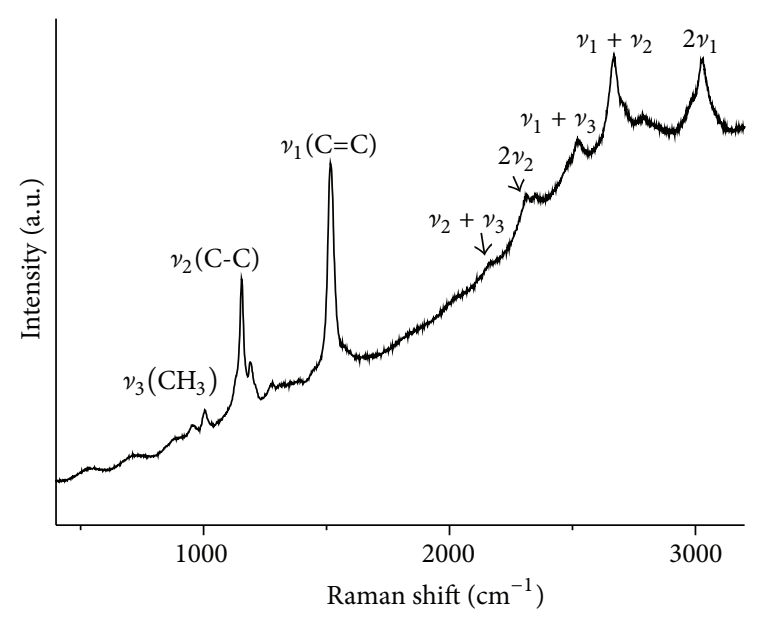

FIGURE 2: Resonance Raman spectrum of canthaxanthin at normal pressure.

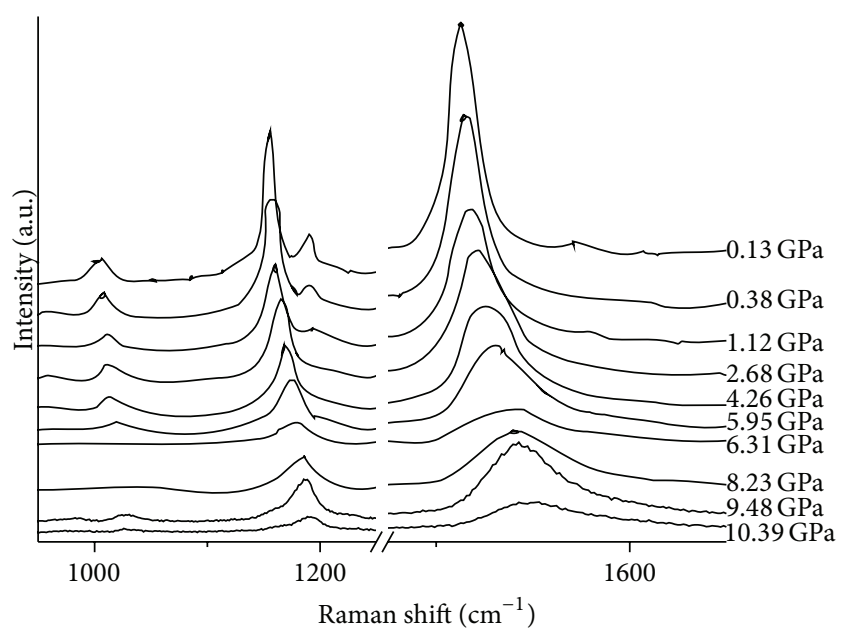

FIGURE 3: Resonance Raman spectra of canthaxanthin at different pressure in the spectral range of $950 \mathrm{~cm}^{-1}$ to $1650 \mathrm{~cm}^{-1}$.

corresponds to the frequency multiplication of the three main bands from $2000 \mathrm{~cm}^{-1}$ to $3100 \mathrm{~cm}^{-1}$ [15].

3.2. Raman Spectra of Canthaxanthin under High Pressure. Figure 3 shows the resonance Raman spectra of canthaxanthin from $950 \mathrm{~cm}^{-1}$ to $1650 \mathrm{~cm}^{-1}$ at different pressures 


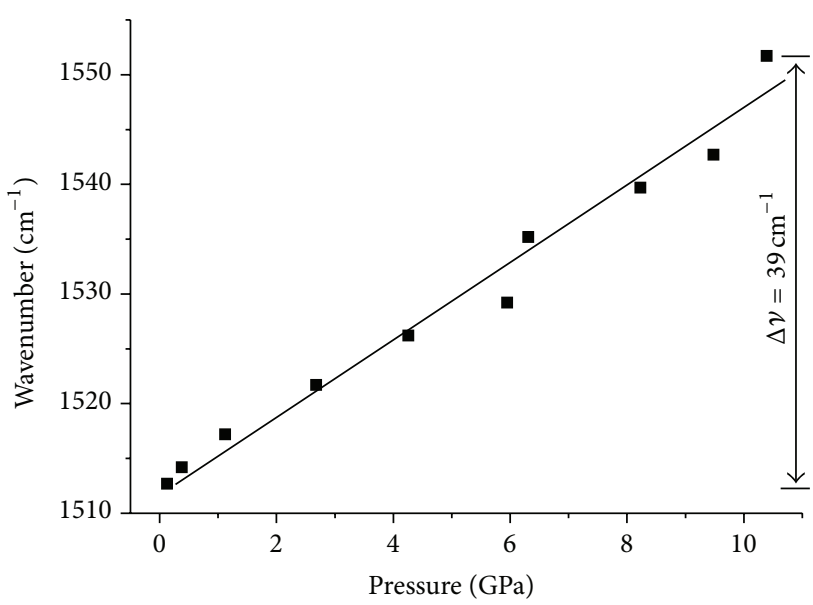

(a)

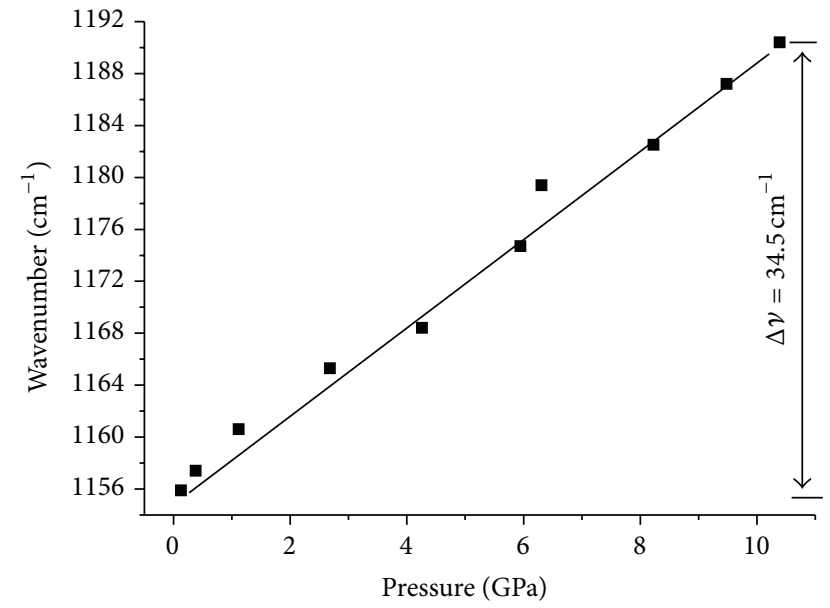

(b)

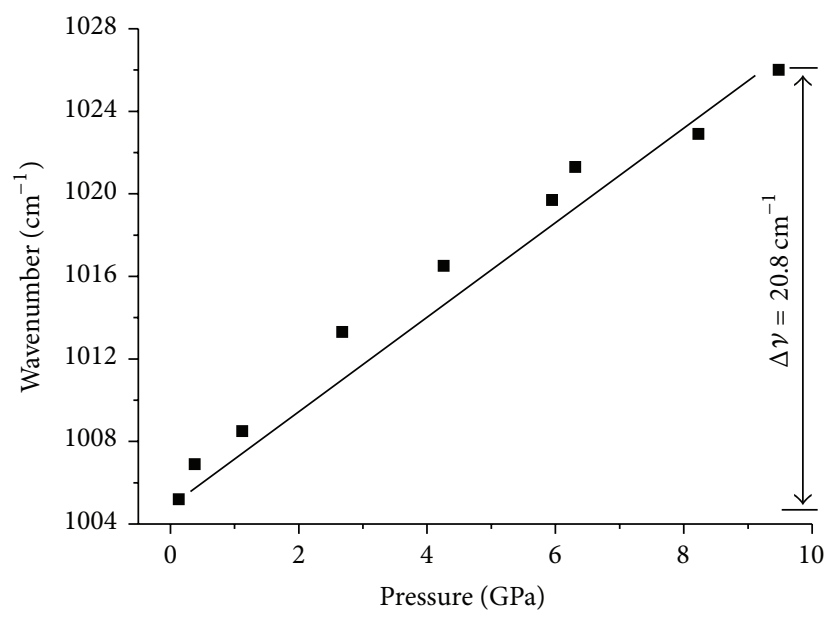

(c)

Figure 4: Pressure dependence of (a) $v_{1}$, (b) $v_{2}$, and (c) $v_{3}$ frequencies of canthaxanthin.

and at room temperature. To simplify our data, we did not indicate the Raman spectra between 1250 and $1475 \mathrm{~cm}^{-1}$ where the Raman band of diamond is unnecessary. The three main bands of canthaxanthin shift gradually toward high frequencies with increasing pressure. It could be explained by the structural distortion of the $\pi$-electrons delocalization. Additionally, the broadening of the three main bands with a loss of intensity was also observed. The broader bandwidth of the three main bands was in accordance with general rules $[12,16]$. When the molecule is compressed, the increased force constant leads to a decreased vibrational cycle. Therefore, the phenomenon resulted from the shorter vibrational relaxation time under high pressure.

In order to systematically study the effect of pressure, the changes of the $v_{1}, v_{2}$, and $v_{3}$ frequencies are plotted versus pressure in Figure 4 . Thus, regression fitting to $v_{1}(\mathrm{C}=\mathrm{C})$, $v_{2}(\mathrm{C}-\mathrm{C})$, and $v_{3}\left(\mathrm{CH}_{3}\right)$ and pressure was conducted, and fitting linear functions are expressed as follows:

$$
\begin{array}{ll}
\nu_{1}(\mathrm{C}=\mathrm{C})=3.43 P+1512.3 & \left(R^{2}=0.98\right), \\
\nu_{2}(\mathrm{C}-\mathrm{C})=3.29 P+1156.1 & \left(R^{2}=0.99\right), \\
\nu_{3}\left(\mathrm{CH}_{3}\right)=2.16 P+1006.3 & \left(R^{2}=0.99\right) .
\end{array}
$$

These equations suggest that the three main bands of canthaxanthin exhibit good linear relation with pressure.

The same as the case of $\beta$-carotene [14], the rate of increase of the $v_{1}(\mathrm{C}=\mathrm{C})$ frequency is faster than that of the $v_{2}(\mathrm{C}-\mathrm{C})$ frequency for canthaxanthin. This intriguing phenomenon was analyzed in the next subsection. The $\nu_{1}(\mathrm{C}=\mathrm{C})$ frequency of canthaxanthin increases slower than that of $\beta$-carotene, while the $v_{2}(\mathrm{C}-\mathrm{C})$ frequency of canthaxanthin increases faster than that of $\beta$-carotene. Under the same experimental conditions, we can therefore deduce the different behavior of the CC stretching frequency for the two studied carotenoids due to their different chemical structures. Being nonpolar, $\beta$-carotene is randomly distributed in solution resulting in glassy matrix [17] under high pressure. Polar canthaxanthin, which has two carbonyl groups in both rings, may adopt some well-defined preferred orientations [18] forming crystalline-like matrix under high pressure.

Figure 5 shows the resonance Raman spectra of the frequency multiplication of canthaxanthin at different pressures. Compared with Figure 3, which shows the Raman spectra of the fundamental frequency of canthaxanthin, Figure 5 shows that the patterns of changes in fundamental frequency and frequency multiplication of canthaxanthin under high 


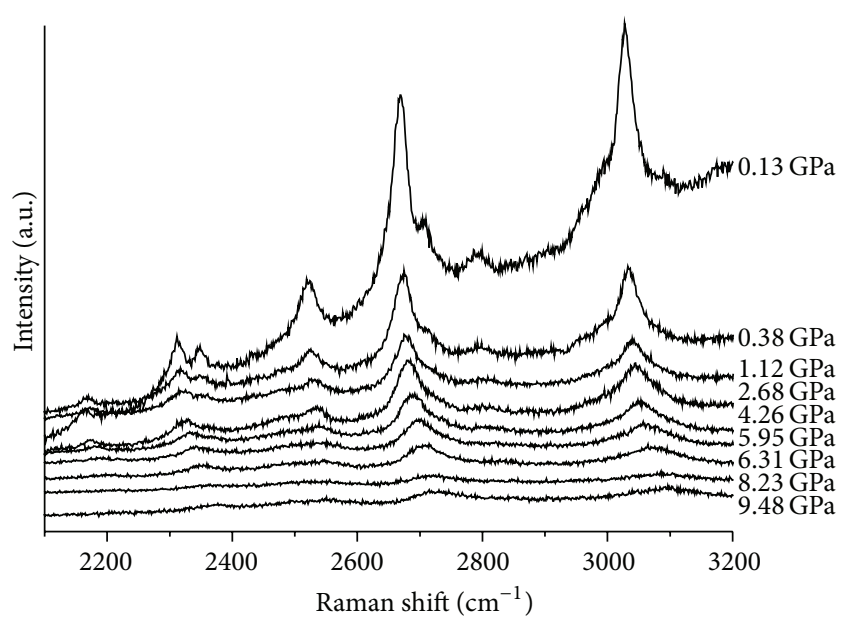

FigURE 5: Raman spectra of canthaxanthin at different pressure in the spectral range $2100-3200 \mathrm{~cm}^{-1}$.

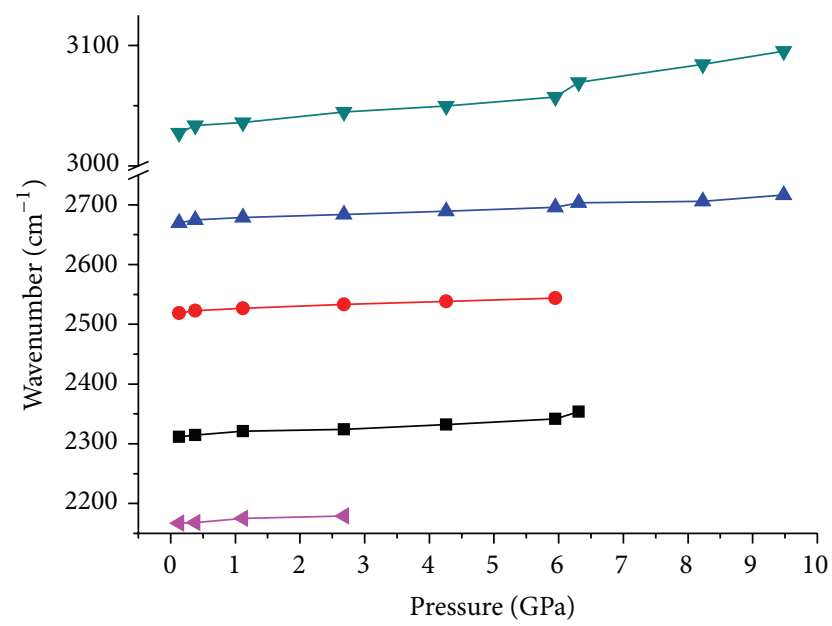

FIGURE 6: Pressure-induced Raman shift of various modes of canthaxanthin in the spectral region of $2100 \mathrm{~cm}^{-1}$ to $3200 \mathrm{~cm}^{-1}$.

pressure are consistent. With increasing pressure, the five Raman bands shift to high frequencies, and the broadening of the bands with a loss of intensity. It could be seen from Figure 6 that the rate of increase of the overtone $2 v_{1}$ frequency is faster than that of another frequency multiplication as pressure increases.

3.3. Effect of High Pressure on the Molecular Structure and $\pi$-Electron Delocalization of Canthaxanthin. Canthaxanthin is a conjugated molecule; furthermore, $\pi$-electrons delocalization affects molecular structure, free radical spin, electronic excitation state, nonlinear optics, and physicochemical properties of canthaxanthin. As such, the influence of high pressure on molecular structure and $\pi$-electrons delocalization of canthaxanthin was analyzed. Figure 4 shows that the slope for the $v_{1}$ is larger than $v_{2}$, which indicates that the pressure effect on the $\mathrm{C}=\mathrm{C}$ stretching vibration is more sensitive than that on the $\mathrm{C}$ - $\mathrm{C}$ stretching vibration. However, this is inconsistent with the general idea that the
$\mathrm{C}=\mathrm{C}$ bonds are compressed more hardly than $\mathrm{C}-\mathrm{C}$ bonds. This can be explained by taking account of the structural distortion of the $\pi$-electrons delocalization. It is well-known that the $v_{1}$ frequency in polyene can be used to monitor the degree of $\pi$-electrons delocalization/conjugation through the $\pi$-electron system [19]. The $\mathrm{C}=\mathrm{C}$ stretching vibration frequency is decreased by the increased conjugation length of polyene, because, with the increase of the conjugation length, the $\pi$-electrons delocalization tends to equalize the CC bond lengths. Accordingly, for the distorted canthaxanthin, which has a relatively shorter conjugation length of the conjugated chain, the frequency of the $v_{1}$ band shifts to high frequency. Therefore, our experimental result obtained with canthaxanthin is a downward shift of the $v_{1}$ frequency under our experimental condition, and this means that the degree of $\pi$-electrons delocalization/conjugation through the $\pi$-electron system is changed for the distortion of molecular configuration.

3.4. Phase Change in Canthaxanthin under High Pressure. The resonance Raman spectra are recovered after the compression-decompression cycle. This indicated that canthaxanthin does not undergo irreversible modifications under our experimental condition, that is, pressure below 10.39 GP and $514.5 \mathrm{~nm}$ excitation. Therefore, canthaxanthin has no evident phase change in the specified pressure range used in this study.

\section{Conclusion}

In this study, in situ high-pressure Raman spectroscopy was applied to obtain the resonance Raman spectra of canthaxanthin. At room temperature, all of the Raman bands of canthaxanthin increase and reach a high wavenumber without undergoing a phase change as pressure increases from normal to $10.39 \mathrm{GPa}$. The results showed that the degree of the $\pi$-electrons delocalization in canthaxanthin molecule is weakened under high pressure. Therefore, this study provides an experimental basis of the photoelectric mechanism of canthaxanthin in photoelectric devices.

\section{Conflict of Interests}

The authors declare that there is no conflict of interests regarding the publication of this paper.

\section{Acknowledgments}

This study was supported by the National Natural Science Foundation of China (Grant nos. 11364027, 21363013, 11564031 and 11004252), the Fundamental Research Funds for the Central Universities (no. 14CX02162A), Key Laboratory of Integrated Exploitation of Bayan Obo Multi-Metal Resources Independent Subject (Grant no. BO-13-004), and Inner Mongolia University of Science and Technology Innovation Fund (Grant nos. 2012NCL001, 2012NCL005, and 2014QNGG09). 


\section{References}

[1] T. Goswami, S. K. Karthick Kumar, A. Dutta, and D. Goswami, "Control of laser induced molecular fragmentation of n-propyl benzene using chirped femtosecond laser pulses," Chemical Physics, vol. 360, no. 1-3, pp. 47-52, 2009.

[2] F. Momicchioli, G. Ponterini, and D. Vanossi, "First- and second-order polarizabilities of simple merocyanines. An experimental and theoretical reassessment of the two-level model," Journal of Physical Chemistry A, vol. 112, no. 46, pp. 11861-11872, 2008.

[3] D. A. Jose, A. D. Shukla, G. Ramakrishna, D. K. Palit, H. N. Ghosh, and A. Das, "Physicochemical and photophysical studies on porphyrin-based donor-acceptor systems: effect of redox potentials on ultrafast electron-transfer dynamics," Journal of Physical Chemistry B, vol. 111, no. 30, pp. 9078-9087, 2007.

[4] E. M. J. Johansson, T. Edvinsson, M. Odelius et al., "Electronic and molecular surface structure of a polyene-diphenylaniline dye adsorbed from solution onto nanoporous $\mathrm{TiO}_{2}$," Journal of Physical Chemistry C, vol. 111, no. 24, pp. 8580-8586, 2007.

[5] J. Li, J. K. Tomfohr, and O. F. Sankey, "Theoretical study of carotene as a molecular wire," Physica E, vol. 19, no. 1-2, pp. 133$138,2003$.

[6] V. E. de Oliveira, H. V. Castro, H. G. M. Edwards, and L. F. C. de Oliveiraa, "Carotenes and carotenoids in natural biological samples: a Raman spectroscopic analysis," Journal of Raman Spectroscopy, vol. 41, no. 6, pp. 642-650, 2010.

[7] M. Di Valentin, S. Ceola, G. Agostini et al., "Pulse ENDOR and density functional theory on the peridinin triplet state involved in the photo-protective mechanism in the peridininchlorophyll a-protein from Amphidinium carterae," Biochimica et Biophysica Acta-Bioenergetics, vol. 1777, no. 3, pp. 295-307, 2008.

[8] S. M. Budy, S. Suresh, B. K. Spraul, and D. W. Smith Jr., "Hightemperature chromophores and perfluorocyclobutyl copolymers for electro-optic applications," Journal of Physical Chemistry C, vol. 112, no. 21, pp. 8099-8104, 2008.

[9] T. W. Hagler, K. Pakbaz, K. F. Voss, and A. J. Heeger, "Enhanced order and electronic delocalization in conjugated polymers oriented by gel processing in polyethylene," Physical Review B, vol. 44, no. 16, pp. 8652-8666, 1991.

[10] L. C. Hoskins and V. Alexander, "Determination of carotenoid concentrations in marine phytoplankton by resonance Raman spectrometry," Analytical Chemistry, vol. 49, no. 6, pp. 695-697, 1977.

[11] H. Hayashi, T. L. Brack, T. Noguchi, M. Tasumi, and G. H. Atkinson, "Vibrational relaxation in carotenoids in vivo and in vitro: picosecond time-resolved anti-Stokes resonance Raman spectroscopy," Journal of Physical Chemistry, vol. 95, no. 18, pp. 6797-6802, 1991.

[12] W.-L. Liu, Z.-R. Zheng, R.-B. Zhu et al., "Effect of pressure and solvent on Raman spectra of all-trans- $\beta$-carotene," Journal of Physical Chemistry A, vol. 111, no. 40, pp. 10044-10049, 2007.

[13] W. L. Liu, Z. R. Zheng, Z. F. Dai et al., "Effect of $\beta$-ring rotation on the structures and vibrational spectra of $\beta$-carotene: density functional theory analysis," The Journal of Chemical Physics, vol. 128, pp. 124501-1-124501-7, 2008.

[14] N.-N. Wu, S.-L. Ouyang, and Z.-W. Li, "Study on the effect of pressure on the molecular structure and $\pi$-electron delocalization of $\beta$-Carotene by Raman Spectroscopy," Spectroscopy and Spectral Analysis, vol. 33, no. 9, pp. 2429-2432, 2013.
[15] S.-L. Ouyang, Z.-W. Li, Y.-Z. Chen, Z.-W. Men, N.-N. Wu, and C.-L. Sun, "Spectra of linear polyene molecule-canthaxanthin," Chemical Research in Chinese Universities, vol. 27, no. 4, pp. 661663, 2011.

[16] K. Wang, D. Duan, R. Wang et al., "Stability of hydrogenbonded supramolecular architecture under high pressure conditions: pressure-induced amorphization in melamine-boric acid adduct," Langmuir, vol. 25, no. 8, pp. 4787-4791, 2009.

[17] A. Andreeva, I. Apostolova, and M. Velitchkova, "Temperature dependence of resonance Raman spectra of carotenoids," Spectrochimica Acta-Part A: Molecular and Biomolecular Spectroscopy, vol. 78, no. 4, pp. 1261-1265, 2011.

[18] N.-N. Wu, Z.-W. Li, J.-Y. Liu, and S.-L. Ouyang, "Effects of the structural order of canthaxanthin on the Raman scattering cross section in various solvents: a study by Raman spectroscopy and ab initio calculation," Chinese Physics B, vol. 21, no. 10, Article ID 103101, 2012.

[19] R. J. Weesie, J. C. Merlin, J. Lugtenburg, G. Britton, F. J. H. M. Jansen, and J. P. Cornard, "Semiempirical and Raman spectroscopic studies of carotenoids," Biospectroscopy, vol. 5, no. 1, pp. 19-33, 1999. 

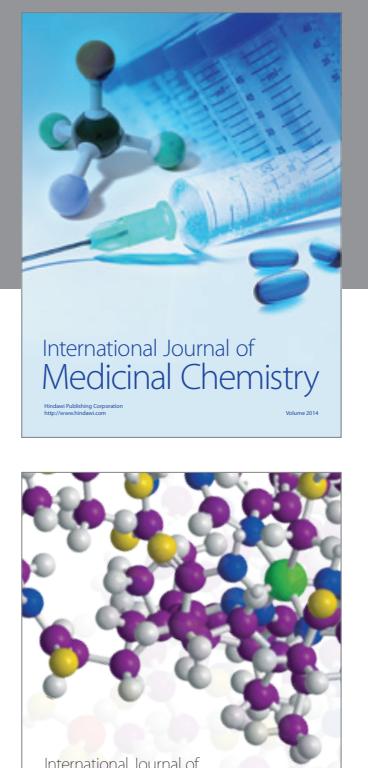

\section{Carbohydrate} Chemistry

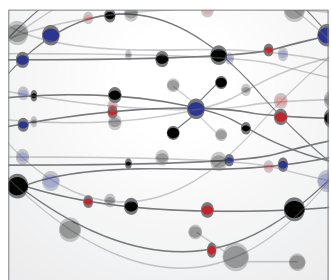

The Scientific World Journal
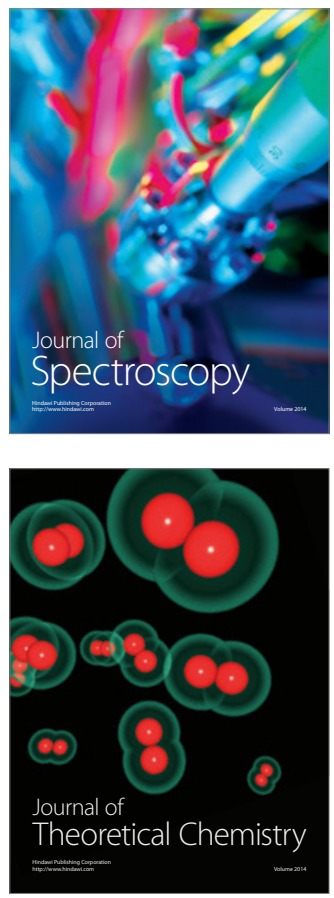
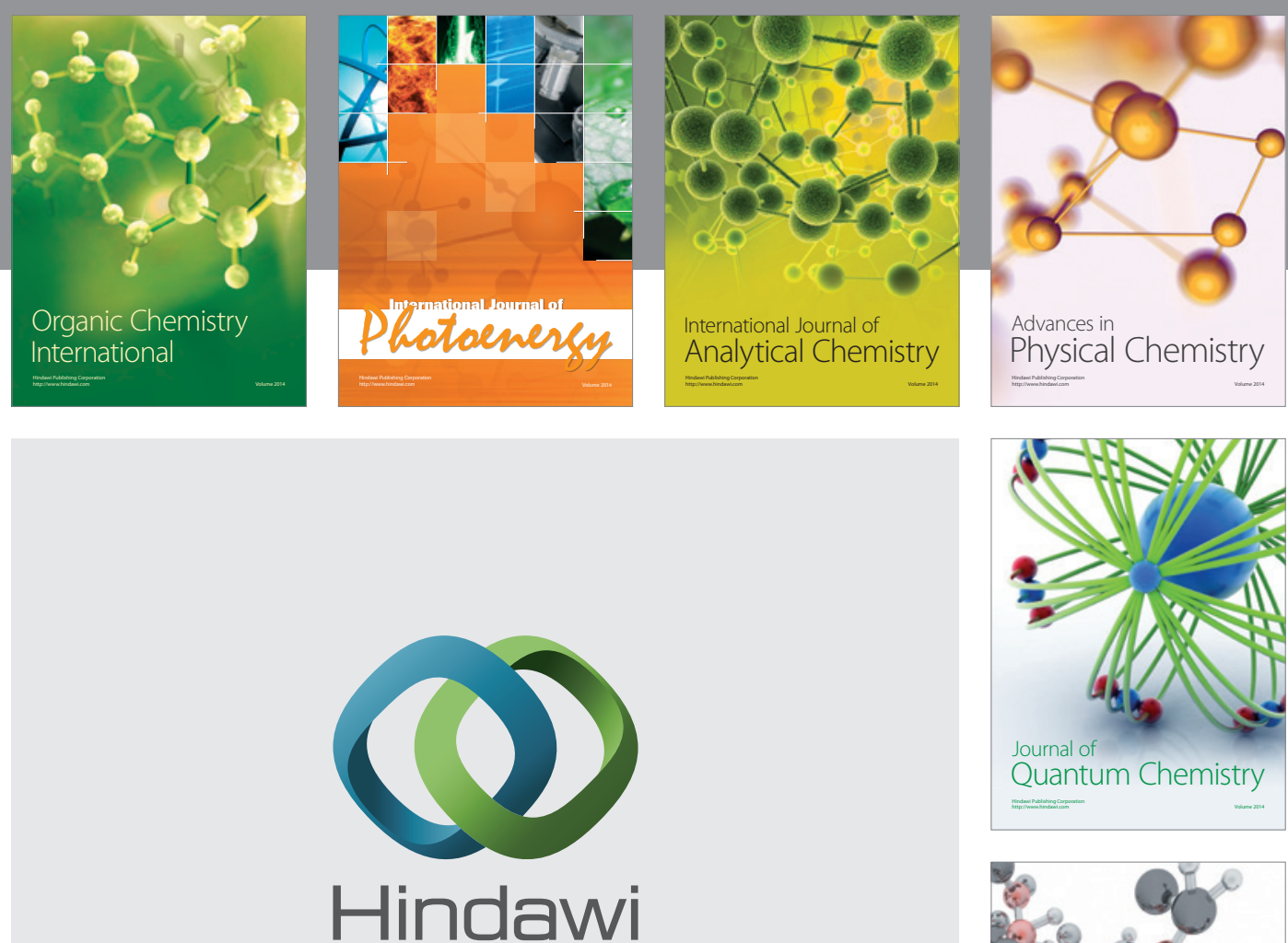

Submit your manuscripts at

http://www.hindawi.com

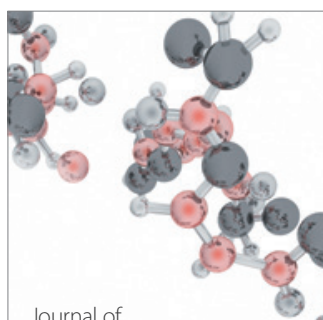

Analytical Methods

in Chemistry

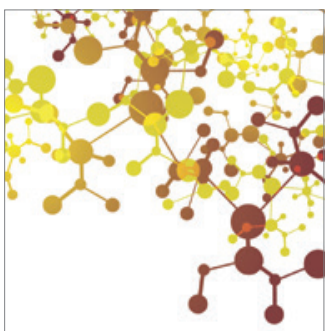

Journal of

Applied Chemistry

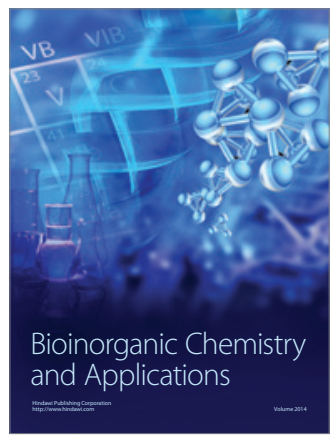

Inorganic Chemistry
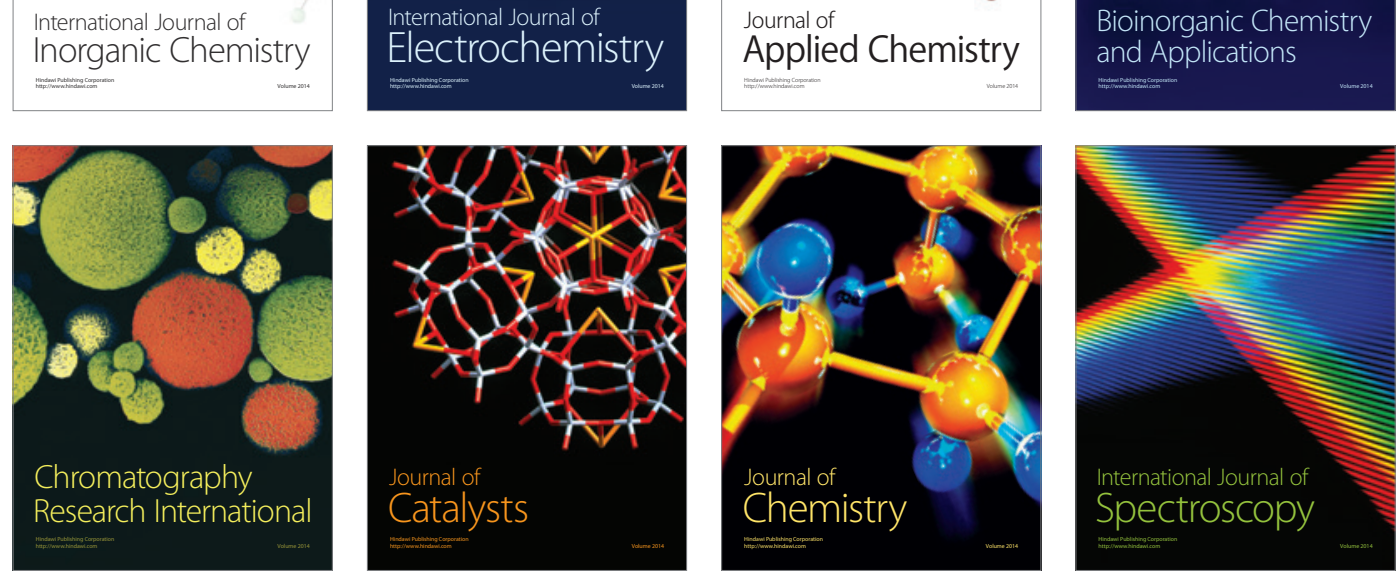